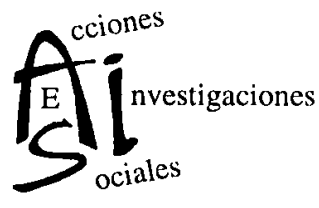

\section{Formación y empleo: empresas, organizaciones y requerimientos cualificacionales}

\author{
David Pac Salas \\ DEPARTAMENTO DE PSICOLOGÍA Y SOCIOLOGÍA \\ UNIVERSIDAD DE ZARAGOZA
}

El objetivo en el artículo es presentar los resultados de una investigación sobre los requerimientos futuros a la fuerza de trabajo. Se trata de estructurar los enfoques discursivos de los actores sociales que participan e intervienen en los procesos de trabajo: empresarios, sindicatos y trabajadores. Se adopta una concepción de las cualificaciones como producto social en el que inciden tanto las condiciones económicas y técnicas como las políticas y sociales. Los requerimentos de cualificación a la mano de obra en las empresas son fruto de una construcción social.

Palabras clave: requerimientos, empresas, empleo, formación. 


\section{Formación y empleo: empresas, organizaciones y requerimientos cualificacionales \\ David Pac Salas}

\section{Introducción}

Las relaciones entre formación y empleo perfilan un panorama de indudable importancia y trascendencia política y social. Desde los postulados de la teoría de capital humano (Schlutz, 1961;Becker, 1964), la educación, probablemente, nunca ha dejado de ser un tema de interés destacado para los responsables económicos, políticos y sociales.

En las sociedades capitalistas avanzadas, caracterizadas por una permanente transformación y mutación en todos los ámbitos, una de las parcelas fundamentales donde los cambios parecen más rápidos, es en el mundo económico y empresarial. Algunas estimaciones sobre los empleos y puestos de trabajo, hechas en el ámbito de la Unión Europea, revelan que en un plazo de diez-quince años, un 75-85 por \% de los puestos de trabajo alterarán sustancialmente sus contenidos, señalando la dificultad de precisar cuáles van a ser las nuevas tareas a desempeñar. Ello requiere que nos preguntemos ¿qué nuevos empleos?, ¿qué nuevas formas organizativas? ¿qué puestos de trabajo?, ¿qué tareas? ¿qué conocimientos ${ }^{1}$ ? Algunos autores,

1 Es una temática recurrente en la literatura especializada. Por citar algunos ejemplos recientes, en el ámbito de la sociología, el estudio ¿Sirve la formación para el empleo?, llevado a cabo por Grup d'Estudis Sociològics sobre la Vida Quotidiana i el Treball -QUIT- (2000).Otro interesante trabajo, centrado en los fenómenos de formación y empleo, es el libro Formación y empleo, coordinado por Felipe Sáez (2000), resultado de un programa de investigación de la Fundación Argentaria 
como Homs y Prieto (1991: 218 y ss), señalan que es un fenómeno que se convierte en casi explosivo «si se considera que está teniendo lugar en un brevísimo espacio de tiempo. Lo viejo y lo nuevo se entremezclan sin dar tiempo a una reflexión suficiente y a una consolidación de los procesos».

El objetivo en el artículo es presentar los resultados de una investigación sobre los requerimientos a la fuerza de trabajo en el futuro ${ }^{2}$. Se trata de estructurar los enfoques discursivos de los individuos que participan e intervienen en los procesos de trabajo. La propuesta es adoptar una concepción de las cualificaciones como producto social en el que inciden tanto las condiciones económicas y técnicas como las políticas y sociales. Los requerimientos de cualificación a la mano de obra en las empresas son fruto de una construcción social (Homs y Prieto, 1991; QUIT, 2000). Nos interesa indagar como los diferentes actores, que construyen esos requerimientos, interpretan su papel activo en el devenir cualificacional, y por tanto, son protagonistas con sus acciones $u$ omisiones en ese devenir.

En primer lugar se hace un breve repaso de las diferentes posturas relativas al concepto de cualificación. En un segundo apartado, se señala la metodología y el diseño de los grupos de discusión. A continuación se analizan los discursos de los diferentes actores sociales, y por último se presentan unas conclusiones del estudio.

\section{Empresas, organización del trabajo y requerimientos de cualificación}

La estrategia empresarial explica cómo el proceso reorganizador, en las últimas décadas, proviene de las propias empresas, y es que «la contracción e inestabilidad de los mercados y la diversificación de la producción pusieron de manifiesto las debilidades del taylorismo-fordismo» (Brunet y Belzunegui, 2000:

2 Este texto tiene su base en una parte del Informe de Investigación, para el programa FORCEM, bajo el título «Estudio sobre la prospectiva de demandas de cualificación profesional para las PYMES», dirigido por el profesor José Ramón Solans Solano de la Universidad de Zaragoza. Zaragoza, diciembre de 1997, inédito. 
146). Algunos autores señalan que estos cambios nos llevan a unas nuevas formas de organización de las empresas: tanto desde el punto de vista técnico como organizativo. Entre los apologistas del fin del fordismo y los pesimistas del cambio, nosotros señalamos que se están produciendo algunas metamorfosis ${ }^{3}$ importantes en el mundo empresarial y social. Aunque sin cuestionar los principios últimos de la organización taylorista: la división del trabajo, la economía de los tiempos y la especialización de las tareas ${ }^{4}$. Sostenemos que debemos rechazar la idea mecanicista de un futuro plenamente determinado, aunque esto no implica situarse en el polo opuesto, es decir un futuro plenamente indeterminado. El futuro está co - derterminado por el pasado, nuestras acciones ya realizadas, y por tanto, lo que vendrá no está determinado de un modo definitivo y absoluto, y hoy todavía podemos actuar. Y el grado de determinación/indeterminación será medido por las posibles influencias de nuestras acciones. Solamente aquellos procesos en los que no podemos ejercer influencias indican un futuro determinado. Si un día podemos influir en estos procesos, conociendo sus leyes de comportamiento y generando los medios técnicos para variarlos, habremos creado nuevas posibilidades de futuro, y lo posible habrá dejado de ser lo único posible, lo necesario.

En el mundo complejo de las organizaciones empleadoras se viene observando unos cambios con relación a qué cosas deben de saber hacer, qué tipo de habilidades sociales deben poseer, los diferentes componentes de la fuerza de trabajo. Partiendo del eclecticismo existente en torno a modos diferentes de racionalización en el trabajo, desde su versión técnica (innovaciones tecnológicas), como en su versión organizativa, se ha suscitado, en las últimas décadas, una fuerte polémica a como ha repercutido estos cambios en la cualificación de los trabajadores. Los contenidos concretos que recubren las nociones de

3 Como, señala Castel (1997:13 y ss) «en estos tiempos de cambio e incertidumbres, en los que el pasado se oculta y el futuro es indeterminado, teníamos que movilizar nuestra memoria para tratar de comprender el presente».Cuando hablamos de metamorfosis, dialéctica de lo igual y lo diferente: identificar las transformaciones históricas de este modelo, subrayar lo que sus principales cristalizaciones traen a la vez de nuevo y de permanente, así sea bajo formas que no permiten reconocerlas de inmediato.

4 Véase Martín Artiles (1999) 
racionalización de la organización del trabajo, educación, formación, cualificación, requerimientos son ahora muy diferentes hoy en día de lo que lo eran hace 20 años.

De manera muy esquemática, se han dado dos posturas básicas: la escuela regulacionista y la institucionalista.Los regulacionistas consideran que los cambios forman parte de un nuevo régimen de acumulación que consideran dominante (Coriat, Boyer, Aglietta y Palloix).Mientras que los institucionalistas, analizan las transformaciones bajo el modelo de especialización flexible, que son las estrategias empresariales de adaptación al nuevo entorno surgido tras la crisis económica de los setenta-ochenta (cuyos principales exponentes son Piore y Sabel). Entre estas perspectivas es donde debemos inscribir el debate sobre la cualificación.

¿Que entendemos por requerimientos de cualificación? Para la comprensión y análisis de estos requerimientos cualificacionales los diferentes autores centran su punto de interés en diversos aspectos. Vamos a señalar algunos elementos. Para Fernández Steinko, A. (1996: 288), vendrán determinados por los puestos de trabajo, así señala que «los requerimientos de cualificaciones dependen de la cantidad y la calidad de las tareas incorporadas a los puestos de trabajo. Estas últimas, a su vez, son el resultado de la estrategia de modernización y la estructura organizativa derivada a ella. No es de extrañar, por tanto, que la primera valoración que hacen de las cualificaciones realmente requeridas dependa en gran parte de las mencionadas estrategias seguidas así como de las expectativas puestas en dichas estrategias, la propia valoración subjetiva de la función de la fuerza de trabajo, etc.».

Otros autores centran su interés en el análisis del proceso de trabajo, es el caso de Lope (1995: 55), «la razón es que la cualificación no puede analizarse más que donde los trabajadores realizan sus funciones laborales». Por tanto, teniendo en cuenta la cualificación efectiva, es decir, "la que es real y efectivamente requerida y ejercida en el proceso de trabajo y por las exigencias empresariales específicas, relacionados a su vez con la posesión de conocimientos y capacidades de actuación por parte del trabajador adecuados a aquellos requerimientos».

Distinguiendo la formación»para» y «en» el trabajo puesto que las innovaciones organizativas y el sistema técnico «hacen obso- 
letos los criterios a través de los que se articulan las cualificaciones». Al introducirse cambios y complejidad a las tareas, «los conocimientos que exigen y el grado de control y de intervención sobre el propio trabajo». Es decir, «la formación <para> acceder al empleo debiera atender los nuevos requerimientos, al tiempo que los ya ocupados deben obtener nuevo contenidos formativos (formación <en> el empleo) para adaptarse a las nuevas situaciones de trabajo» Lope y Artiles (1993), QUIT (200)

Por último, como hemos señalado anteriormente, vislumbramos un proceso social en la construcción de los requerimientos cualificacionales «la presunción de que los cambios de cualificación constituyen un proceso social complejo de intereses, estrategias y políticas, en el cual juegan un papel destacado las posiciones y acciones de los distintos agentes que participan en él» (IESA 1987: 76).Y entendemos la cualificación como un proceso social, «el concepto de cualificación entendido como proceso social. Los requerimientos de cualificación, tanto funcionales como sociales, independientes de personas concretas, para el cumplimiento de ciertas tareas del trabajo, que funcional y posicionalmente se engloban en un puesto de trabajo.» IESA (1987: 77)

Planteando en estos términos el asunto, optamos por un concepto más abierto, más dinámico, ya que, se pudiera hablar de cualificación refiriéndose a las capacidades para adaptarse al todo de las condiciones de trabajo, incluido su movimiento. Conceptualización que va más lejos de las más habitualmente usadas, se trata de capacidades suficientes para llevar a cabo cierta actividad intelectual, de pensamiento, de conocimiento de fenómenos propios de la naturaleza de las cosas (físicos, químicos,...), y de cierta capacidad de intervención (prácticas) » ${ }^{5}$. Por tanto hablaríamos de «recualificación» cuando se viera incrementado el uso que hace el empleador de estas capacidades de la fuerza de trabajo.

\section{Cuestiones metodológicas}

Para ello hemos optado por analizar los enfoques discursivos de los en las posiciones de los principales protagonistas: los em-

5 Véase Solans, Pac, Navarrete (1997) 
pleadores y los trabajadores.Los empresarios (entendiendo como tal a aquellas personas que ejercen el control de la empresa en general, y de la mano de obra en particular, sean o no los propietarios de ellas), se han visto en este siglo dentro de un entorno en constante cambio. Por tanto, los empresarios han tenido, tienen y tendrán que adaptarse a un entorno socioeconómico mutable y variable a través de distintas estrategias. Las estrategias empresariales se configuran en relación con otras empresas y diferentes mercados, sobre la base de los sucesos del presente y en base a las transformaciones que dichos sucesos acarreen. Dentro de la estrategia empresarial, que permite la adaptación y la supervivencia de la empresa, se encuentra la estrategia empresarial acerca de la fuerza de trabajo. La posición de los trabajadores se dividió, por un lado, en trabajadores cuya actividad exclusiva es la actividad sindical.Y por otro lado, en trabajadores cuya actividad se desarrolla en los procesos de trabajo de las empresas.

Por el carácter abierto de los discursos era necesario una metodología que permitiese aprehender el sentido de las opiniones, imágenes, actitudes de los empleadores y trabajadores respecto a cómo vislumbran esa realidad más o menos mediata. Se optó por la realización de 7 grupos de discusión buscando la representatividad discursiva del tejido empresarial de Aragón.

Diseño de los grupos de discusión.

El diseño de los Grupos de Discusión es el momento más «artesanal». En este trabajo «artesanal» se intenta representar a las distintas posiciones en la estructura de relaciones laborales. Por un lado, la posición del empresarios, y por otro, los trabajadores, y la posición que ocupan los sindicatos como representantes de los trabajadores. Nos interesaba indagar en las diferentes percepciones, que se vislumbran sobre las estrategias en la acción sindical.A su vez hemos querido reflejar, como señala, la literatura existente ${ }^{6}$, las diferencias y semejanzas, que aparecen en los diferentes actores dependiendo su posición en uno u otro sector: producción de bienes o de servicios.

Aunque como señalan autores como Castells, (1997:233), la noción de servicios puede ser ambigua o engañosa. La categoría servicios es utilizada como «cajón de sastre», donde ubicar,

6 De los trabajos recientes J. Rosa Marrero Rodríguez, ST núm. 26 «Semejanzas y diferencias en la producción de bienes y servicios». 
aquellas actividades «que abarca todo lo que no es agricultura, minería, construcción, servicios públicos o fabricación».Algunos de los intentos de definirlos mediante algunas características intrínsecas , como su intangibilidad, opuesta a materialidad de los bienes, han quedado definitivamente desprovistos de significado por la evolución de la economía informacional.

\section{Actores}

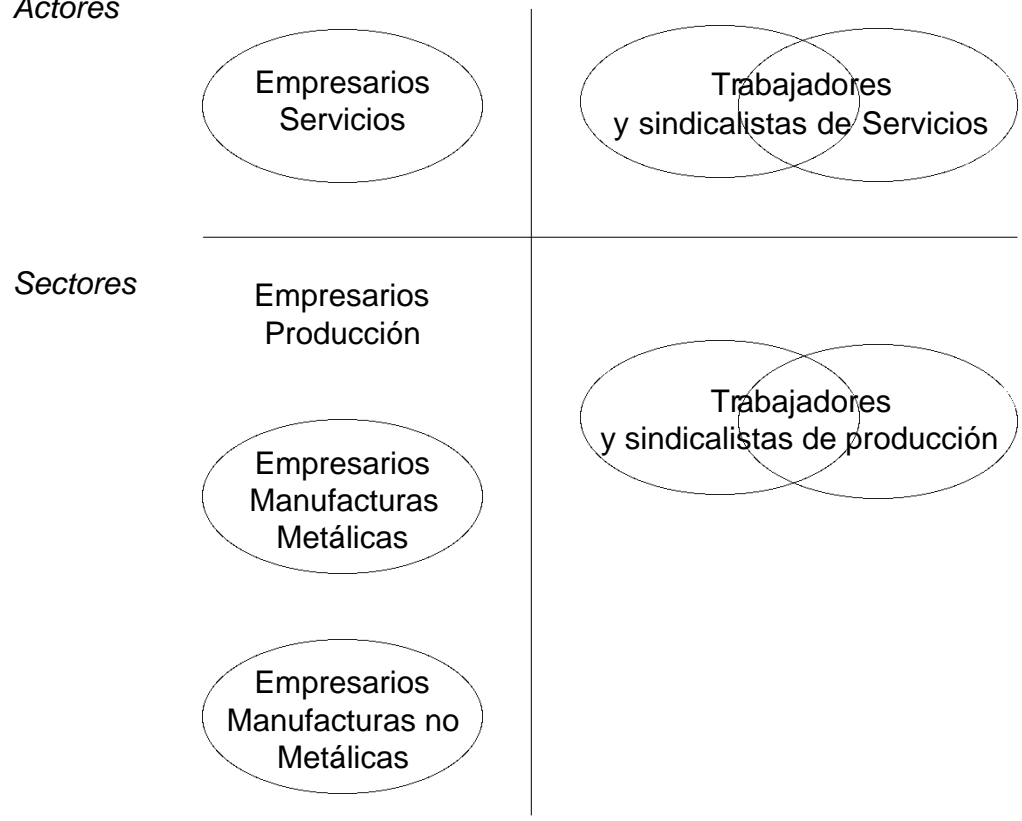

Esquema 1.Diseño de los grupos

\section{Anáisis de los grupos de discusión}

\subsection{Discurso empresarial}

\subsubsection{Empresarios servicios}

Este grupo se plantea las necesidades de requerimientos desde la posición de cual es su ventaja competitiva en un sector en que todos ofrecen prácticamente el mismo producto, al- 
canzando un consenso en el que se establece «el cliente como objetivo", y las reacciones de éste marcaran la estrategia.

Una estrategia en la que la capacidad de respuesta es básica, atendiendo los cambios de comportamiento y de gustos del cliente.Todo es tan duradero o tan efímero como desee el cliente, de tal forma que el cliente es quien define el producto, y por tanto el proceso de su elaboración, ya no vale encerrarse en el laboratorio y definir nuevos productos.Se impone la «cultura del cliente», el tratamiento, ya no diferenciado, individualizado del cliente.La dependencia del cliente, del individuo concreto es total, todas las técnicas son puestas a disposición en la forma que desea el cliente, el valor añadido, lo es, si así lo percibe el cliente.

En esta situación, el trabajador adquiere una nueva dimensión, el trabajo, el comportamiento concreto del trabajador es fundamental. El trabajador, en el desarrollo de su trabajo es la imagen de la empresa, esta es la segunda dependencia, cuestión que se hace más dolosa asumir debido al nivel de prestigio que tienen ciertas ocupaciones de este sector; y de buen o mal grado se trata de asumirla, y ahí topamos con la mentalidad, la forma de ver las cosas del empresario.

A su vez, la competencia en términos de costes lleva a una organización del trabajo que requiere la flexibilidad de tareas, para lo que se hace imprescindible que todos los trabajadores tengan una formación global de todas las tareas diferentes que se llevan a cabo. Aunque la flexibilidad de tareas no siempre es posible por las reticencias de trabajadores de categorías altas, o algunas otras categorías más específicas, con más tradición, $p$. e. los cocineros.

Conseguir llevar a cabo este tipo de estrategias, en las que se es muy dependiente de los trabajadores, lleva consigo la necesidad de que los propios trabajadores se impliquen en la empresa, dándole a conocer datos, el propio proyecto empresarial.

Las modalidades puestas en práctica o disponibles por los empresarios son variadas y no llegan a establecer consenso sobre las más adecuadas, pues todas tienen sus defensores y sus detractores, ya sea facilitando la promoción, a través de cursos, o devolviéndole la dependencia con la seguridad en el empleo.

Al fin y a la postre, el tema de la implicación se convierte en un tema de actitudes, que conjuntamente con otras serán la base de los requerimientos en un futuro no demasiado lejano, 
que para algún miembro del grupo se puede convertir en requerimiento suficiente, aunque otros lo descartan rotundamente como requisito suficiente.

Estas dificultades, en cuanto al cumplimiento de los requerimiento son hoy más tangibles, pues es propio de una situación de cambio, y lo que se requiere es de la sociedad, en su conjunto, unos nuevos valores que permitan la adaptación al cambio.

Si estos son los requerimientos esenciales, también lo son otro tipo de requerimientos, otro tipo de cualificaciones para las que son precisos unos conocimientos, que pueden ser aprendidos en un modelo formativo, modelo que deberá plantearse una formación de base amplia que ayude a pensar, a encontrar respuestas y soluciones a las cosas, y una formación más práctica que ayude a saber desarrollar lo que se piense. Y que aporte elementos necesarios para saberse relacionar, saber tratar a los demás.

En los servicios como en cualquier otra actividad las cosas siguen cambiando, y si se quiere tener la capacidad de respuesta, que se hace necesaria para la estrategia, se aprecia en este sector un grave inconveniente por el propio horario de trabajo, lo que llevará a replantearse la jornada, y la posible formación en horario de trabajo.

En este grupo se plantea de forma especial dos colectivos de trabajadores importantes en el sector, ya sea por los requerimientos específicos que se necesitarán, los mandos intermedios; ya sea por la diferente concepción de su función, los vendedores.

\section{Los mandos intermedios}

En este caso parece necesaria una cualificación que pueda llevar a la implicación al resto de los trabajadores, para lo que necesitara disponer de conocimientos de la globalidad del proceso para poder comprender el proyecto de empresa, conocimientos sobre costes, actitudes muy marcadas hacia la calidad y saberse comunicar con los demás, conocimiento amplio del lenguaje, capacidad de síntesis.

\section{El vendedor}

Sobre las características que debe requerir un vendedor tampoco se establece acuerdo, configurándose dos posiciones. El vendedor que es bueno lo es en todos los terrenos. El vendedor para ser bueno debe conocer a fondo el producto que vende y al cliente al que se lo vende. 
No obstante, para este caso la necesidad que se ve más fundamental para el futuro es que se produzca un cambio en cuanto a la percepción que de él se tiene.

\subsubsection{Empresarios manufacturas metálicas}

Los representantes de los empresarios del metal se plantean, de cara a poder detectar sus requerimientos para el futuro, un escenario, que continuando tendencias, aparece atravesado por el ahorro de costes en un mercado muy competitivo, de tal forma que en las sociedades occidentales solamente pervivirán aquellos procesos que no puedan ser asumidos por la mano de obra de terceros países, en los que su coste es mucho menor, y lo esencial de las sociedades más avanzadas industrialmente será diseñar y dirigir los procesos, tareas éstas que emergerán con un nuevo cuño y que exigirán un alto nivel de conocimientos y con capacidad de decisión.

En este escenario la pequeña y mediana empresa jugará un papel, pero un papel diferente al actual. En el terreno de la producción estará sometida a un proyecto, formando parte de una red de empresas que participarán en ese proyecto.

El terreno de la distribución se configurará como el elemento flexible de la gran empresa para rebajar costes.Aparecen dos posiciones semidefinidas en cuanto a cual va a ser la estrategia de las empresas respecto de los productos y de los clientes.Una más orientada al cliente que entrará a definir el producto, y otra más orientada a la elaboración de productos diferenciados de otros del mismo uso para los que será preciso encontrar compradores.

Pero para ambos casos se requerirán personas con alta formación tanto tecnológica como financiera.Y en cualquier caso lo que no será compatible con las estrategias será la forma tradicional de pensar la empresa como un bien patrimonial más.

Para poder llevar adelante este tipo de estrategias la organización de la empresa requerirá fundamentalmente en el terreno del proceso de trabajo concreto flexibilidad de tareas, y trabajo en equipo que posibilite la movilización de conocimientos colectivos, de forma que la comunicación de saberes y experiencias conlleve un salto cualitativo en el conocimiento global.

Se da por supuesta la necesidad de que la práctica totalidad de los trabajadores tengan conocimientos de idiomas y de informática, aunque más en aquellos puestos o niveles que implican 
relación con personas de otros países, aunque en este caso más que de conocimientos se podría decir perfecto conocimiento de otros idiomas. Informática e idiomas que entrarán a formar parte de una formación humana y científica básica, que aún siendo elemental posibilite al trabajador para luego incorporar a ella elementos más específicos.

La formación humana supone en su contenido una serie de valores culturales de acuerdo con la sociedad global que se nos presenta, en la que se van a poner en contacto personas pertenecientes a diferentes culturas locales con las que deberemos ser capaces de trabajar, de colaborar.

La formación científica básica deberá incluir temas relativos a qué es una empresa, qué es el mercado, las tecnologías, elementos que luego se va a encontrar en la vida. De forma especial esta formación básica requerible deberá contener conocimientos de las herramientas disponibles en el momento, de manera que pueda utilizarlas, y con carácter especial conocimientos de lógica, que sirvan para racionalizar los procesos, los comportamientos.

Para conseguir esto, parece que el grupo vería la necesidad de plantearse también el sistema educativo, en el sentido de la lejanía que hay entre la formación teórica y su aplicación práctica en el mundo del trabajo, que posibilitase la resolución de los problemas concretos que se presentan en la empresa.

No obstante no hay que dejarlo todo en manos del sistema formativo externo a la empresa, pues será la propia empresa quien facilite la formación específica necesaria para realizar las tareas concretas, que aporten habilidades y ayuden a recorrer el camino entre lo abstracto aprendido fuera y lo concreto de las tareas.

Esta formación que corresponde a la empresa será una formación continuada, pues las tecnologías, los procesos cambian permanentemente. Esto requiere necesariamente del trabajador la capacidad de aprender, para lo que tiene que tener unos conocimientos anteriores que posibiliten el aprendizaje posterior.

Pero, parece que el trabajador disponga de estas posibilidades de aprender no es garantía suficiente, pues es requisito indispensable una actitud favorable, que parta de un conocimiento de que en el más inmediato futuro va a necesitar nuevos conocimientos, y cuando ya los haya adquirido, las cosas cambiaran de nuevo, y necesitará otros. 
La actitud positiva a aprender lo nuevo forma parte de la actitud positiva hacia el cambio, de la capacidad de adaptación al cambio que la empresa requiere para poder llevar a cabo sus estrategias de flexibilidad, que contienen variaciones en los procesos de trabajo, en las tareas. Esto deberá ser interiorizado por los trabajadores de forma que sea un valor más dentro del acerbo de valores que configuren su personalidad.

El grupo distingue como específicos de cara a los requerimientos necesarios cuatro grupos de funciones en las que habría encuadrados distintos trabajadores

\section{La función de mantenimiento}

Que, aunque similar a lo que en otro tiempo pudiera haberse llamado «manitas», precisamente esta distancia temporal la hacía de cara al futuro muy diferente, fundamentalmente los trabajadores responsables de esta función deberán disponer de una extensa gama de requerimientos que les lleven a tener un conocimiento lo más amplio posible del proceso global.

\section{La función de recursos humanos}

Basada necesariamente en la nueva organización de trabajo en equipo, que sea capaz de generar actitudes positivas hacia la movilización de conocimientos colectivos y hacia la calidad.

\section{Los trabajadores de producción}

Los requerimientos de formación para este grupo deberá permiterles especializarse en un conjunto de tareas.

\section{La función comercial}

Con capacidad de analizar el mercado, los clientes y saber conocer el producto en toda su amplitud.

\subsubsection{Empresarios manufacturas no metálicas}

Para poder plantearse los requerimientos que les serán demandados a los trabajadores en el futuro, se plantean la situación de la empresa en ese futuro, que, a su juicio, va a estar determinado fundamentalmente por las variaciones del mercado y de la técnica.

La empresa del futuro se caracterizará por estar inmersa en una red de relaciones con otras empresas en aras de conseguir la elaboración de productos destinados al mercado. A su vez, la 
evolución técnica y del mercado de consumo conllevará unas relaciones más directas con el consumidor final, con el cliente.

Esa nueva forma de articular los procesos de trabajo tendrá lugar incluso en aquellas ramas de la producción en las que la división del proceso de trabajo entre empresas, generando diferentes centros de trabajo desde los que se participa en el producto final, llevan un cierto «retraso histórico», como el caso de la construcción.

De ahí que la función de relaciones con el conjunto de empresas que configuren dichas redes, y con los consumidores últimos se adivine como una función con alta hegemonía.

Los propios procesos de trabajo van a estar sujetos a variaciones emanadas de los nuevos valores de los que se está dotando la sociedad civil, y a exigencias cada vez más intensamente vividas por los trabajadores en cuanto a sus condiciones de trabajo, sus condiciones de vida en el lugar de trabajo.

Estas van a ser nuevas tareas que deberán desarrollarse en las empresas, conjuntamente con las tareas de adaptación de nuevas tecnologías, emanadas de la innovación informática, a las realidades concretas de los procesos de trabajo en cada empresa. Ahora bien, no parece que se tratará tanto de la adaptación, aunque también, de elementos técnicos, como de la construcción propia de tecnologías posibles, en base a la situación en cada momento del proceso científico-técnico.

Estas nuevas relaciones hacia afuera y hacia dentro supone un cambio en los valores, un cambio cultural en el mundo de la empresa, favorecido por los nuevos valores que incorporan los nuevos trabajadores, cambio cultural que pone en cuestión las estructuras organizativas jerárquicas. Dando paso a nuevas estructuras organizativas, menos jerárquicas, en las que las relaciones entre los trabajadores conllevarán relaciones de poder más equivalentes, con mayor comunicación entre ellos sobre temas relativos al trabajo concreto, posibilitando la transferencia de experiencias y conocimientos individuales, generando conocimientos colectivos.

Se tratará de generar equipos de trabajo en los que estarán integrados el conjunto de trabajadores de la empresa, de modo que se posibilite una cierta participación en las decisiones de cómo se desarrollan las tareas y su contenido, movilizando así otras capacidades que disponen los trabajadores, independien- 
temente del lugar ocupado en el proceso de trabajo. La probabilidad de que tenga lugar este suceso será mayor, cuanto mayor sea la formación de los trabajadores, lo que, a su vez, incrementará las posibilidades de que la empresa pueda llevar a cabo las estrategias diseñadas.

No obstante, esto sólo será posible consiguiendo la implicación de los trabajadores en la empresa, y un cambio en los valores de todos los que desarrollen alguna actividad en la empresa, será precisa una nueva forma de la entender la «empresa-organización» por todos sus miembros.

Esta nueva forma organizativa requerirá de los trabajadores unos conocimientos sobre relaciones interpersonales, sobre los otros, y más específicamente para quienes tengan mayores cuotas de responsabilidad conocimientos sobre funcionamiento de grupos, técnicas de motivación, etc., que en todos los casos, estos conocimientos deben integrarse en los módulos de formación básica. A su vez, el que los trabajadores alcancen un cierto nivel de conocimientos técnicos posibilitará una mejora en las condiciones de trabajo, mediante una disminución de riesgos laborales. Siguiendo con la formación, parece que se debe conseguir integrar formación teórica y formación práctica.

Se reconoce a la empresa como una entidad en permanente movimiento, con necesidad de introducir variaciones, por lo que los futuros trabajadores deberán, en su periodo formativo, habituarse a reconocer las distintas posibilidades de actuación, y el probable impacto de cada una de ellas en la globalidad del mundo de relaciones de la empresa, de cara a desarrollar la capacidad de toma de decisiones.

Como requerimientos básicos y universales para la mayoría de los puestos de trabajo se nombran conocimientos de informática y de idiomas, en el primer caso porque la informática va a estar presente en todos los momentos del proceso de trabajo, y en el segundo por la creciente relación con empresas, clientes de entornos lingüísticos diferentes.

Si bien se percibe la necesidad de una formación básica amplia y con contenidos de conocimientos diferentes, parece que lo característico de la formación va a ser su continuidad a lo largo de la vida, sobre todo en la adquisición de conocimientos específicos, más propios de las actividades concretas de las distintas empresas. 
Tres son las tipologías de trabajadores para las que parece habrá requerimientos más diferenciados en el futuro: trabajadores de producción, técnicos y mandos intermedios.

El trabajador de producción, al que se le requerirá una cierta formación técnica, pues las tareas que se le encomienden, dejarán de ser tareas simples para pasar a manejar máquinas herramientas un tanto complejas. Y que en una organización flexible del proceso de trabajo se le requerirán capacidades para el manejo de diferentes máquinas-herramientas a lo largo de las jornadas de trabajo.

En el caso de los técnicos, el requerimiento fundamental consistirá en capacidades de dar respuesta a las incesantes variaciones del mercado, nuevas situaciones, nuevos problemas, nuevas soluciones. Estas tenderán a incrementar la calidad de la producción y la productividad por unidad de tiempo, pero que en absoluto pueden ser ajenas a la relación coste/beneficio.

Para los mandos intermedios se ven como imprescindibles amplios conocimientos sobre relaciones interpersonales y funcionamiento de grupos.

\subsection{Discurso sindical}

\subsubsection{Sindicatos de producción}

El grupo representante de los sindicatos de producción vislumbran un cambio en los modos de producción actuales, en las demandas de consumo y el mercado de trabajo que modificarán sustancialmente los requerimientos de cualificación del futuro.

Las estrategias empresariales se basarán en la transformación de los modelos productivos actuales. Reseñan como escenario de futuro de los procesos actuales una sustitución de mano de obra directa por la contratación o arrendamiento de servicios. Ven el futuro en «el trabajo en casa a través del manejo del ordenador».

Otro elemento clave, en las estructuración del discurso del grupo, es la transformación de las condiciones de mercado de bienes. La internacionalización de la economía, y en el caso español, la europeización, nos llevan a la necesidad de conocer idiomas. Existe un consenso unánime en todos los miembros del grupo, como requerimiento esencial, transversal, para toda la 
fuerza de trabajo, «el idioma, el bilingüismo, lo entiendo como una formación base casi de ciudadano». La interiorización de dicho valor idiomático ha sido muy potente en los sujetos de este grupo.

Por otra parte, las modificaciones en los procesos de producción, que conlleva una automatización de los procesos y la necesidad de conocimiento de informática en los niveles de producción. La característica esencial es la informatización de los procesos «no la informática para ser usada a nivel personal sino la informática para lo que da de sí en los procesos productivos». La automatización requerirá especialistas en materias cada vez más concretas con el «riesgo» de la pérdida de los profesionales u oficios convencionales: soldadores, torneros... Debido a las dificultades de la empresas medianas y pequeñas a modernizarse y a las series cortas en algunas piezas (altos costes en capital fijo de la innovación tecnológica) se requieren en la actualidad estos oficios clásicos que requieren largos períodos de aprendizaje «debido a la industria de CNC se pierden algunos oficios».

Otra valor básico, la necesidad de especialización, con la dificultad de definir los requerimientos, vislumbrando una disminución de los universitarios y un aumento de la presencia de la formación profesional. Por tanto, analizan un cambio en la orientación formativa, ya que «hasta ahora formación genérica no específica». Aunque parece una contradicción entre «un especialista y alguien que lo hace todo». No acaba de definir el grupo esa paradoja.

La necesidad de producción de calidad alta, es para este grupo esencial, puesto que la competitividad conlleva a una mayor profundidad en los temas de calidad y elaboración de los bienes con una normativa estricta.

Se vislumbra una necesidad de una formación continua para reciclarse en los distintos puestos de trabajo (formación para toda la vida) y debido a la necesidad de polivalencia de los trabajadores.

La conclusión seria un trabajador con una cualificación amplia, especializado en una tarea más o menos sencilla, una tarea no muy compleja, estamos hablando de trabajadores de producción con unos comportamientos flexibles, "elásticos» o «moldeables, adaptables con una actitud positiva hacia el cambio». Mediante la introducción de nuevas tecnologías se necesi- 
tará continuamente aprender más cosas y también de cara a las nuevas formas de trabajo: subcontratación de otras empresas, variaciones dentro de las diferentes empresas y las relaciones en cuanto a la posición que ocupan unas empresas en el proceso global de elaboración de un bien o servicio. Esta posición de la empresa marca los diferentes requerimientos de formación para sus respectivos trabajadores.

Algunas actividades que se desarrollaran por las nuevas necesidades de consumo son las relativas a cuestiones de domótica (edificios inteligentes), así como las telecomunicaciones (cable o fibra óptica), electrónica analógica y digital, así como empresas de cableados. Por tanto estas áreas representan para este grupo las actividades con mayores posibilidades de expansión y por tanto se necesitarán conocimientos en dichas materias.

En cuánto a las nuevas profesiones que se requerirán estarían relacionadas con la conservación del medioambiente «al ecologismo, el medioambiente, etc.", así como expertos en calidad total. Uno de los profesionales con mayor expansión son el personal de mantenimiento que ven una «superespecialización, cada vez las máquinas son más complejas, toda la automatización de las fábricas son más complejas, las máquinas son más complicadas, automatismos...». Por último señalan la transcendencia que tendrán algunas profesiones relacionadas con las cuestiones de seguridad y prevención de riesgos «debido a la entrada en vigor de las nuevas reglamentaciones y normativas».

\subsubsection{Sindicatos de servicios}

Los representantes del grupo señalan, en primer lugar, que el futuro del sector estará condicionado por las variaciones en la estructura social de la sociedad actual y futura. Esta disposición de la estructura social determinará la aparición de nuevas necesidades sociales, que en parte deberán ser satisfechas por el sector servicios, principalmente por los que se denominan sector de servicios sociales.

La mujer y envejecimiento de la población. Las principales variaciones están teniendo lugar con respecto a la diferentes posición que ocupa la mujer en el mercado de trabajo, lo que provoca, un vacío en las funciones desempeñadas tradicionalmente por la mujer, la reproducción social. Tanto el cuidado de los hijos, 
debido al trabajo, como el cuidado de ancianos son necesidades que se deberán satisfacen dentro del sector servicios.

La aparición de actividades de consumo relacionadas con el «tiempo libre», cultura, ocio, conllevaran la aparición de personas con conocimientos específicos en estos ámbitos.

Un elemento esencial, que el grupo asume, es la implantación de las nuevas tecnologías en su trabajo. Aunque señalan algunos aspectos positivos o negativos en su implantación. No llegan a cuantificar si supondrán más aspectos negativos o positivos.

La implantación de la informática en todos los ámbitos y consideran que el sector servicios ha incidido de manera especial, los procesos manuales han sido sustituidos por la generalización de los procesos informáticos.

La formación será eminentemente tecnológica y necesaria; en ocasiones, las nuevas tecnologías son utilizadas sin la necesaria preparación, por tanto, el aprovechamiento de dichas innovaciones no consiguen su máxima extensión.

Señalan tres previsiones futuras de formación a saber: idiomas, consideraciones ético-profesional y factor humano.

Son conscientes de la apertura de nuestra sociedad al exterior y de la confluencia de múltiples culturas entre las que existe un intercambio. El conocimiento de idiomas es imprescindible para establecer relaciones con esas otras culturas.

En lo que se refiere a las consideraciones éticas señalan que en la actualidad se adolece de una preparación concreta en el campo deontológico. Detectan problemas a la hora de poner en práctica la obligación del secreto profesional.

Detectan la necesidad de una formación que de prioridad al factor humano. Lo que significa recibir no una formación centrada exclusivamente en la mecánica, sino que valore, de modo especial, la necesidad de cuidar el aspecto humano. En el sentido de calidad en la atención a los usuarios del sector servicios.

Finalmente los integrantes del grupo especifican una serie de aptitudes y actitudes que deberán ser potenciadas por los trabajadores en el futuro.

El individuo va a tener que desarrollar una serie de capacidades que le permitan adaptarse a un entorno que varía constantemente en el espacio y en el tiempo:

- Capacidad de intervención, decisión y autonomía 
- Capacidad de realizar un trabajo interdisciplinar, un trabajo en equipo

- Capacidad de ser polivalente; que tenga conocimiento de su profesión y al mismo tiempo sea capaz de desenvolverse en otros ámbitos.

- Capacidad de iniciativa, de relacionar unos conocimientos con otros, de comunicarse.

- Capacidad de reciclaje.

Y para desarrollar todas estas capacidades será indispensable que el individuo mantenga una actitud positiva hacia el cambio en todos los órdenes: social, económico y cultural.

Por tanto, perciben la necesidad de formación continua de los trabajadores de una manera "exponencial», puesto que día a día se requerir una rotación profesional.Así como las exigencias de complementariedad ya que, para este grupo, cada vez necesitarán realizar tareas complementarias.

\subsection{Discurso de los trabajadores}

\subsubsection{Trabajadores del sector servicios}

La imagen "común» es un sector en expansión y con gran cantidad de cambios, cuya característica principal es su rapidez. Aparece en sus discursos una necesidad de adaptación a esos cambios y para ello deben de potenciarse una serie de capacidades en los trabajadores. Estas capacidades tienen que venir dadas por una formación amplia y sólida para posteriormente poder especializarse en una actividad del sector servicio, en un producto-servicio, en el puesto de trabajo concreto.

La posición que ocupa al final del proceso de elaboración de un producto-servicio, fundamentalmente conlleva una característica consustancial: la relación directa con los clientes. En el grupo, se produce una sobrerepresentación de la actitudes el "saber estar", ocupando un papel central en sus discursos: capacidad de comunicación, actitudes comportamentales (saber sonreír, don de gentes), capacidades para las relaciones sociales, etc.

En cuanto a la concreción de elementos o requerimientos de cualificación profesional en un futuro se vislumbran necesidades 
formativas en conseguir personas con capacidades para la venta, impartiendo materias o enseñando estos «saberes», aunque deben de tener, a su vez, conocimientos técnicos sobre el producto-servicio que venden.

Se observa la necesidad de algunos conocimientos transversales, que los trabajadores, en general tiene que conocer y utilizar:

- Mecanismos y herramientas para captar información general y específica para cada sector.

- Informatización del sector y por tanto conocimiento en informática.

- Necesidad de idiomas.

- Mayor preparación en materias relacionadas con lo humano (Filosofía y Letras).

Así como algunos conocimientos específicos destacados, la prevención de riesgos laborales, una cuestión que se considera de un interés primordial, especialistas dentro del rama de los seguros (ejemplo árbitros de fútbol).

En cuanto, a las actitudes de los trabajadores, se deben de tratar de trabajadores obedientes, «que se dejen mandar». En el grupo se identifican las trabajadores formadas con mayor facilidad para integrarlos en la empresa, con menor conflictividad, «una persona que tiene formación es mucho más dúctil y manejable que una persona sin formación». Como ya hemos señalado, la relación entre productor-usuario/cliente condiciona de manera importante las cosas que deben hacer los trabajadores de este sector.

Por tanto, como eje central, los requerimientos cualificacionales deberían ir ligados a construir personalidades predispuestas hacia la relaciones sociales, que requieren el "saber estar» y el «saber relacionarse con los otros». Por tanto, potenciar las capacidades humanas, las actitudes comportamentales y capacidades para las relaciones sociales. Se requerirá trabajadores que tengan elementos de empatía hacía los otros.

Para este grupo, el trabajador de futuro es el vendedor, cuestionando el tratamiento que se da en nuestro país, contraponiéndolo al prestigio que tienen EE.UU, llamándole ejecutivo comercial. Por tanto apuestan por «dignificar» la profesión de vendedores desarrollando verdaderos especialistas comercia- 
les. La necesidad de formación en este campo, la necesidad de un «instituto» donde se pudiese formar especialistas «en captación, ejecutivos comerciales».

\subsubsection{Trabajadores de sector producción}

Los requerimientos de cualificación en el futuro son percibidos a través de las variaciones en los procesos productivos. Se alinean con la tesis optimista del futuro de las cualificaciones, así perciben, que se van a exigir, cada día más, una mayor capacidad de iniciativa de los trabajadores los cuales serán por lo general más cualificados. Se produce en el grupo una exaltación a cerca de como serán los profesionales del futuro «muy, muy cualificados».

Se refieren a la necesidad de que los trabajadores sean más polivalentes, algunos de los cuales lo identifican con «manejable». Pero critican la falta de reconocimiento manifiesto por parte de la empresa de esas nuevas competencias.

A su vez en la formación necesaria se observan la importancia de los conocimientos o habilidades personales, así como las habilidades humanas a base de formación. También se destaca la posibilidad de formación a través de cursos, reseñando que en la actualidad, existe mayor facilidad de que se forme. Lo fundamental es una cultura básica amplia humanas (pre-trabajo). La base es una formación cultural amplia más una profesionalidad de oficios básicos (artesanos) con una formación profesional teórico/ práctica.

En cuanto, a la reestructuración por sectores ven con optimismo el sector servicios, particularmente el sector turístico, destacando como un oficio de futuro los cocineros. En industria, se señala la exigencia de mayor calidad en la elaboración de los productos, mayor formación técnica, mayor organización teniendo en cuenta aspectos relativos a los métodos y tiempos en la organización del trabajo. trabajo métodos y tiempo, etc..

La necesidad de conocimiento de la empresa se echa en falta en este grupo sobre todo porque «lo que se conoce se asume mejor», para que tengan sus razonamientos lógicos por que ocurre las cosas», « en el producto se nota la calidad del trabajo»

A su vez, la necesidad de potenciar la capacidad de abstracción de los trabajadores, así observan en el eje jóvenes/mayores una mayor facilidad para aprender, cuyo conocimiento es más fácil.Critican la parcelación del trabajo «el trabajo lo hemos 
dividido demasiado", con la dificultad de entender el proceso de trabajo en toda su extensión.

Por otra parte, se observa, la necesidad de gente sin formación para las cadenas, no hace falta FP2, la gente del campo puede realizar las tareas sencillas. Por tanto, además de una mayor formación, en las empresas de futuro observan como algunas tareas no hace falta mucha cualificación, sino que más bien serán tareas sencillas, rutinarias, etc.. Aunque el grupo no precisa en que consistirán esas tareas poco «cualificadas».

En el grupo, se señala, como los empresarios han logrado interiorizar a los propios trabajadores como contrincantes:jóvenes y mayores.

Un aspecto esencial, en el futuro, será la integración de tareas en algunos trabajadores. La figura profesional de futuro, para este grupo, es los trabajadores de «reparación» o mantenimiento con un amplio abanico de conocimientos: informática, hidráulica, mecánica. Ven una reagrupación de las tareas de producción «quitando» parcelas a las funciones de mantenimiento y administración. Así en un futuro, los propios trabajadores de la máquina realizaran pequeños arreglos, mantenimiento preventivo, etc. Las máquinas facilitan cada vez más el trabajo y lo hacen más sencillo.

Los jóvenes, que por ejemplo, han aprendido con máquinas de control numérico, con conocimientos abstractos, tienen dificultades para llegar a lo concreto, lo que llevará a la pérdida del oficio clásico. Por ello, se necesitarán profesionales de oficio, aunque apuntan que serán necesario en un número pequeño.

Por tanto, como profesionales que destacan para el futuro son: trabajadores de mantenimiento, comerciales, y trabajadores de producción que tendrán algunas tareas integradas.

\section{Estructuración discursiva ante las variaciones de requerimien- tos de cualificación}

En este apartado se presenta un análisis sistemático de los enfoques discursivos básicos sobre los requerimientos a la fuerza de trabajo desde las interpretaciones realizadas sobre las significaciones del debate de los grupos de discusión. 
Las diferentes discursos se estructuran en base a las transformaciones de las condiciones de mercado de bienes y servicios (que en algunos casos vendrán determinados por variaciones de necesidades sociales). ¿Qué cosas se van a producir para cubrir esas necesidades sociales?, ¿qué tipo de productos o de servicios? ¿estandarizados o diversificados?

Un aspecto esencial es definir ¿cómo será la organización del proceso de trabajo en la elaboración de esos bienes o servicios? ¿qué tipo de puestos de trabajo? ¿qué tareas? ¿qué requerimientos cualificacionales tendrá que tener la fuerza de trabajo?

Nuevos mercados / nuevos productos

(bienes o servicios)

Revisión procesos: nuevos/viejos puestos

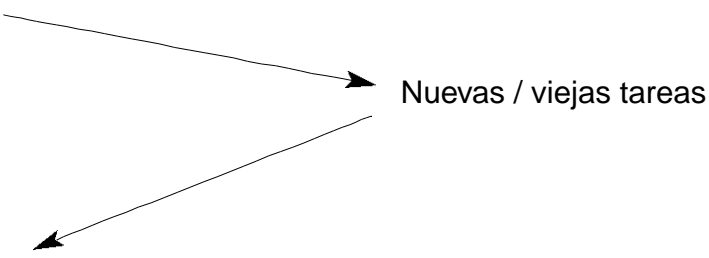

Nuevos/viejos requerimientos

Adiestramiento $\longleftrightarrow$ Formacion

Esquema 2.Metamorfosis de los requerimientos

Las nuevas necesidades sociales conllevaran transformaciones de las condiciones de mercado de bienes y servicios. Estas necesidades vienen marcadas por los cambios en la estructura social, principalmente por el envejecimiento de la población y la 
incorporación de la mujer al mercado de trabajo. Así como, los cambios en los modos de vida, dado el proceso histórico de los diferentes individuos.

En cuanto a los distintos enfoques discursivos podemos destacar dos grandes ejes entre los cuales se articulan los discursos: empresarios/trabajadores y producción/servicio.

Entre las diferentes líneas discursivas se aprecian una serie de coincidencias, en torno a las transformaciones producidas en el empleo y trabajo, si bien, no logran definirlas con precisión, puesto que observan los cambios a gran velocidad y afectando a las estructuras complejas de la organización del proceso de elaboración del producto o bien.

En esas líneas de coincidencia debemos destacar la velocidad de los cambios producidos, la internalización de la economía nos llevará a una necesidad de comunicación, entre los trabajadores de diferentes contextos culturales, en ese nuevo escenario el conocimiento de idiomas, principalmente inglés, se considera una necesidad básica de futuro. Así como los conocimientos de informática, ya sea aplicaciones informáticas en administración como en los diferentes versiones en los procesos productivos. La reestructuración productiva entre sectores vendrá marcada por la evolución hacia una profundización en la terciarización de la economía. Otras coincidencias tendrían que ver con la calidad, importancia de la distribución, requerimientos relacionales o comportamentales, etc (ver las Tablas 1, 2 y 3 , resumen de los principales elementos en las posiciones ideológicas de los grupos)

Estas coincidencias discursivas se ven negadas en la nueva situación sobre el papel que deben de jugar los distintos actores.

\subsection{Transformaciones de las condiciones de mercado de bienes y servicios: papel de la empresa}

Si hoy en día las estrategias empresariales, en el mejor de los casos, estaban construidas sobre los principios de eficiencia global (reducción de costes, mejora de la calidad), sobre principios de innovación (nuevos productos, nuevas características), y en muy pocos casos sobre principios de capacidad de respuesta (atención a diferentes preferencias), rara vez la estrategia estaba construida por la consecución al unísono de las 
tres capacidades eficiencia, innovación y capacidad de respuesta, y las tres capacidades serán necesarias para afrontar el futuro con ciertas probabilidades de éxito. Aquí radica fundamentalmente la necesidad de cambio organizativo.

La posición discursiva de la empresa de futuro vendrá determinada por el tipo de transformaciones de condiciones de mercado de bienes y servicios. Aquí, en primer lugar, debemos destacar, las posiciones ideológicas básicas: empresarios cuya figura discursiva central la empresa, y desde la posición de los trabajadores el elemento principal son los propios trabajadores. Por un lado el discurso empleador, reseña buena parte de sus discursos en el papel que debe jugar la empresa. Frente a esta posición, la posición de la fuerza de trabajo, por lo general, la empresa, o bien, es olvidada por los trabajadores, o tiene connotaciones negativas.

En el discurso empresarial el objetivo primordial es el ahorro de costes (grupo de manufacturas metálicas) y el diseño de la producción, pudiendo obtener mejoras de costes en la distribución. Sin embargo los empresarios de servicios optan por señalar el cliente como objetivo y su preocupación será la búsqueda de estrategias de diferenciación individualizada de productos y fidelización de cliente.

Frente a este discurso la posición de los trabajadores no «perciben» la misma, salvo en el caso, de señalar la falta de información sobre la misma. Señalando, el grupo de trabajadores de producción, el desconocimiento del proceso global de elaboración de un producto, lo cual supone un lastre para la posible introducción de innovaciones de materiales, técnicas, etc.. Así para introducir nuevas formas de organización de la producción, o bien, innovaciones relativas a la mejora de la calidad, estos trabajadores consideran que deben de tener mucha mayor información, para encontrar unos razonamientos lógicos en sus acciones y en las acciones de la empresa.

Entre ambos, los grupos de los sindicatos, participan de la idea de empresa y su reorganización es imaginada mediante el arrendamiento de servicios sustitutiva de la mano de obra directa.Con lo que visto desde el punto de vista sindical puede suponer acrecentar la dificultades de articulación y de maniobra de los intereses sindicales. 
En el terreno de la empresa, parece que se tenderá a profundizar en la especialización, fabricando series muy cortas destinadas a los clientes- individuos concretos, y cuyos productos tendrán una alta velocidad de obsolencencia, incluso en los componentes elementales.

Las relaciones entre empresas cada vez van a ser más acentuadas, configurándose redes de empresas que colaboren en la realización de un producto para el consumo. Estas empresas no requerirán un elevado número de trabajadores, sobre todo de trabajadores directos, aunque si parece que se incrementara notablemente la proporción de trabajadores indirectos.

El principal objetivo de las empresas será ofrecer la posibilidad de elaborar el producto que el cliente desee, que cubra sus necesidades, convirtiéndose el cliente en una parte importante de la empresa, pues va definir la estructura del producto y también el cómo de su elaboración.

El mismo trato con el cliente determinará la posibilidad de su existencia, elaborar o no elaborar productos, de tal forma que la actual área comercial se transformará y adquirirá un peso específico elevado en la empresa, su función esencial consistirá en garantizarle al cliente la posibilidad de elaboración y la calidad del producto deseado, "vender para fabricar», en el mínimo tiempo posible y al coste más bajo posible. Coste y rapidez en el servicio del producto empujan a desarrollar la función logística.

Para desenvolverse en esta nueva situación de mercado, parece que se exige de las empresas una estructura basada en la autonomía de los trabajadores, para lo que será preciso que conozcan lo más posible de todo el proceso de producción, conjuntamente con una implicación de los trabajadores en la empresa. 


\begin{tabular}{|c|c|}
\hline Servicios & Producción \\
\hline $\begin{array}{l}\text { 1. El objetivo de la empresa es «el } \\
\text { cliente». La búsqueda de los } \\
\text { cambios de comportamiento y } \\
\text { de gustos del cliente marcarán } \\
\text { las estrategias futuras de la em- } \\
\text { presa. } \\
\text { 2. Por tanto el compor tamiento } \\
\text { concreto del trabajador es fun- } \\
\text { damental. } \\
\text { 3. Se requerirá una formación glo- } \\
\text { bal de todas las tareas diferen- } \\
\text { tes que se llevan a cabo.Aunque } \\
\text { la flexibilidad de tareas no siem- } \\
\text { pre es posible por las reticencias } \\
\text { de trabajadores de categorías } \\
\text { altas, o algunas otras categorías } \\
\text { más específicas. } \\
\text { 4. Para esa adaptación al cliente } \\
\text { se necesitará una implicación } \\
\text { del trabajador en la empresa. } \\
\text { 5. Y lo que se requiere de la socie- } \\
\text { dad, en su conjunto, unos nue- } \\
\text { vos valores que permitan la } \\
\text { adaptación al cambio. } \\
\text { 6. Y una formación más práctica } \\
\text { que ayude a saber desarrollar lo } \\
\text { que se piense.Y que aporte ele- } \\
\text { mentos necesarios para saberse } \\
\text { relacionar, saber tratar a los } \\
\text { demás. } \\
\text { 7. Señalan un grave inconveniente } \\
\text { por el propio horario de trabajo, } \\
\text { lo que llevará a replantearse la } \\
\text { jornada, y la posible formación } \\
\text { en horario de trabajo. } \\
\text { 8. Las figuras profesionales claves } \\
\text { futuras: mandos intermedios y } \\
\text { vendedores. }\end{array}$ & $\begin{array}{l}\text { 1. El ahorro de costes es funda- } \\
\text { mental así como las fases de di- } \\
\text { seño y dirección de los procesos. } \\
\text { 2. El terreno de la distribución se } \\
\text { configurará como el elemento } \\
\text { flexible de la gran empresa para } \\
\text { rebajar costes, así como inmersa } \\
\text { en una red de relaciones con } \\
\text { otras empresas. } \\
\text { 3. Se requerirán personas con alta } \\
\text { formación tanto tecnológica } \\
\text { como financiera. } \\
\text { 4. La comunicación de saberes y } \\
\text { experiencias conlleva un salto } \\
\text { cualitativo en el conocimiento } \\
\text { global. } \\
\text { 5. Conocer una serie de valores } \\
\text { culturales de acuerdo con la so- } \\
\text { ciedad global. } \\
\text { 6. Será la propia empresa quien fa- } \\
\text { cilite la formación específica ne- } \\
\text { cesaria para realizar las tareas } \\
\text { concretas. } \\
\text { 7. Generar equipos de trabajo en } \\
\text { los que estarán integrados el } \\
\text { conjunto de trabajadores de la } \\
\text { empresa:participación. } \\
\text { 8. La actitud positiva a aprender lo } \\
\text { nuevo forma parte de la actitud } \\
\text { positiva hacia el cambio, de la } \\
\text { capacidad de adaptación al cam- } \\
\text { bio. } \\
\text { 9. Con las tareas de adaptación de } \\
\text { nuevas tecnologías, emanadas } \\
\text { de la innovación informática, a } \\
\text { las realidades concretas de los } \\
\text { procesos de trabajo en cada em- } \\
\text { presa. } \\
\text { 10. Perfiles profesionales: manteni- } \\
\text { miento, recursos humanos, pro- } \\
\text { ducción, comercial. }\end{array}$ \\
\hline
\end{tabular}




\section{Servicios}

1. El futuro del sector estará condicionado por las variaciones de necesidades sociales debidas a cambios en la estructura social de la sociedad actual y futura.

2. Dos elementos clave: papel de la mujer y envejecimiento de la población.

3. Desarrollo de actividades de consumo relacionadas con el «tiempo libre», cultura, ocio.

4. La implantación de las nuevas tecnologías y la reestructuración del trabajo de futuro.

5. Señalan tres previsiones futuras de formación a saber: idiomas, consideraciones ético - profesional y factor humano.

6. Necesidad de profesionales vinculados a cubrir las nuevas necesidades sociales: servicios sociales (tercera edad, infancia), tiempo libre, etc.
Producción

1. El cambio en los modos de producción actuales, en las demandas de consumo y el mercado de trabajo que modificarán sustancialmente los requerimientos de cualificación del futuro.

2. Reestructuración de la producción: como escenario de futuro de los procesos actuales una sustitución de mano de obra directa por la contratación o arrendamiento de servicios.

3. La internacionalización de la economía, y en el caso español, la europeización, nos llevan a la necesidad de conocer idiomas.

4. Los procesos de producción, que conlleva una automatización de los procesos y la necesidad de conocimiento de informática en los niveles de producción.

5. Se requerirá necesidad de especialización, con la dificultad de definir los requerimientos, vislumbrando una disminución de los universitarios y un aumento de la presencia de la formación profesional.

6. La necesidad de producción de calidad alta, es para este grupo esencial, puesto que la competitividad conlleva a una mayor profundidad en los temas de calidad y elaboración de los bienes con una normativa estricta.

7. Perfiles profesionales de futuro: personal de mantenimiento, medioambiente, calidad, seguridad e higiene. 


\begin{tabular}{|c|c|}
\hline Servicios & Producción \\
\hline $\begin{array}{l}\text { 1. Un formación amplia y sólida } \\
\text { para posteriormente poder es- } \\
\text { pecializarse en una actividad del } \\
\text { sector servicio, en un producto - } \\
\text { servicio, en el puesto de trabajo } \\
\text { concreto. } \\
\text { 2. Una característica consustan- } \\
\text { cial: la relación directa con los } \\
\text { clientes. } \\
\text { 3. En el grupo se identifican las } \\
\text { trabajadores formadas con } \\
\text { mayor facilidad para integrarlos } \\
\text { en la empresa, con menor con- } \\
\text { flictividad. } \\
\text { 4. Los requerimientos cualificacio- } \\
\text { nales relativos deberían ir liga- } \\
\text { dos a construir personalidades } \\
\text { predispuestas hacia la relacio- } \\
\text { nes sociales, que requieren el } \\
\text { «saber estar» y el «saber rela- } \\
\text { cionarse con los otros. } \\
\text { Apuestan por «dignificar» la pro- } \\
\text { fesión de vendedores desarro- } \\
\text { llando verdaderos especialistas } \\
\text { comerciales. }\end{array}$ & $\begin{array}{l}\text { 1. La capacidad de iniciativa de los } \\
\text { trabajadores los cuales serán } \\
\text { por lo general más cualificados. } \\
\text { 2. Los trabajadores sean más poli- } \\
\text { valentes, algunos de los cuales } \\
\text { lo identifican con «manejable». } \\
\text { 3. La importancia de los conoci- } \\
\text { mientos o habilidades persona- } \\
\text { les, así como las habilidades hu- } \\
\text { manas a base de formación. } \\
\text { 4. Las funciones de los trabajado- } \\
\text { res de producción se ven incre- } \\
\text { mentadas con la integración de } \\
\text { algunas tareas propias del man- } \\
\text { tenimiento e incluso de tareas } \\
\text { desarrolladas por en la adminis- } \\
\text { tración». } \\
\text { 5. El grupo define como figuras cla- } \\
\text { ves profesionales personal de } \\
\text { mantenimiento, producción, co- } \\
\text { mercial). }\end{array}$ \\
\hline
\end{tabular}

Tabla 3. Posiciones ideológicas de los trabajadores

5.2 Estructura ideológica: el papel de la fuerza de trabajo en la empresa.

En los diferentes grupos la figura a desempeñar por los trabajadores sale reforzada con las nuevas formas de reorganización de los procesos productivos. La fuerza de trabajo, en la práctica totalidad de los grupos pasará a ser más cualificada y deberá poseer más conocimientos. La integración de los trabajadores en la empresa es un componente vital para la empresa de futuro. La implicación de los trabajadores, sobre todo, en las 
empresas dedicadas a actividades de servicios se percibe como uno de los déficits actuales y que deberán corregir en un futuro.

Sobre el papel, para los empresarios, siguiendo planteamientos de la teoría del capital humano, los recursos humanos son el mejor capital de la empresa, la educación y la formación son muy buenos para la empresa y la adaptación de la empresa a los cambios y transformaciones futuras.

En los grupos de discusión de los trabajadores se identifica cualificación con categoría profesional, y su proceso de interiorización es potente. Por tanto, las estrategias de las empresas por incrementar el número de tareas a realizar, o la que es lo mismo, búsqueda de polivalencia en los trabajadores, pasa para los trabajadores, por un aumento de la cualificación. Aunque la empresa requiera de los trabajadores nuevos conocimientos si no son reconocidos como categoría profesional tiene connotaciones negativas.

La empresa de futuro conllevará una búsqueda de la integración de tareas, de algunas de las tareas administrativas en las actividades de fabricación (trabajadores de producción). Un ejemplo, el «jefe de cadena», señalando como ventajas: mejor utilización de la mano de obra mediante la activación de gran parte del tiempo muerto en el sistema automatizado; menores posibilidades de roce por eliminación de demarcaciones profesionales; mayores motivaciones para el trabajo mediante una división en tareas más atractivas y otras razones similares.

De los trabajadores se requerirán diferentes capacidades relativas, a poder establecer relaciones y comunicarse con otros trabajadores, capacidad de comunicar los conocimientos adquiridos con la experiencia, capacidad de diagnóstico y construcción de hipótesis para adelantarse a las disfunciones, capacidad de adaptación en una situación en movimiento permanente, y actitudes positivas respecto a la calidad y al cliente.

Para cumplir con estos requisitos los empresarios buscan titulados a nivel de formación profesional, lo que, hasta hace poco tiempo, chocaba con los deseos de la población adulta y joven, pues los nuevos trabajadores se inclinaban más por titulaciones de nivel universitario, ya que simbolizaba una posición social más elevada. Si bien se sigue manteniendo este simbolismo a nivel de prestigio, en lo concreto del mundo del trabajo, parece que no tienen, la mayoría de las titulaciones, su parangón en 
cuanto a cuantías retributivas y niveles de responsabilidad. Lo que esta acarreando un cambio de actitud hacia la formación profesional.

Estos requerimientos no parece que sea posible alcanzarlos con la actual estructura de especialidades ni de estructuras curriculares. Los empresarios necesitarían planes de estudios mucho más flexibles, en los que se incluyesen, con carácter obligatorio disciplinas que ofreciesen un amplio conocimiento de las teorías científico- técnicas y humanísticosociales.

Los conocimientos a impartir deben experimentar un incremento en las áreas humanístico-sociales, sin por ello descuidar las áreas científico-técnicas, aunque en estas últimas no se hace necesaria una excesiva especialización.Pues la especialización, lo más específico, además de ser muy cambiante, se considera que debe ser alcanzada en el interior de la empresa mediante formación continua.

Pasamos a señalar en forma de conclusiones algunos de los aspectos más importantes debatidos en los grupos y en nuestra estrategia de investigación en torno a las cualificaciones y los diversos requerimientos futuros de la fuerza de trabajo.

\section{Conclusiones}

Como hemos visto a lo largo del artículo, en nuestro planteamiento, los individuos que participan en los procesos de trabajo construyen los requerimientos cualificacionales en el futuro. Por eso, en el proceso social de construcción de requerimientos cualificacionales será muy importante el comportamiento, las acciones y las «omisiones» de los diferentes individuos en la concreción/inconcreción de las mismas.

En concreto, los discursos ideológicos, de los diferentes actores que participan en la construcción social de los requerimientos cualificacionales, vienen marcados por: la posición que ocupa en la estructura de relaciones laborales y por posición en el proceso productivo de elaboración de un bien o servicio, así como por la posición que ocupa en la estructura social y familiar que diferencia a los trabajadores según su poder social de ne- 
gociación, es decir, según el nivel de aceptación de las condiciones de trabajo?.

De todo lo anteriormente dicho se pueden extraer cinco funciones diferenciadas y necesarias para toda actividad económica ${ }^{8}$ : función comercial, función de diseño, función logística, función de producción y función de relaciones humanas.

1. Función comercial: en la que el vendedor dejará de serlo para pasar a ser "perceptor de necesidades», su función será de prediseñador, acotará el producto basándose en el conocimiento obtenido sobre la utilidad que los distintos clientes le otorguen al producto.

2. Función de diseño:su responsabilidad será el diseño concreto del producto o servicio sobre la base de los conocimientos que tenga sobre los posibles diferentes elementos simples, y sus propiedades, que puedan integrarse en el producto o servicio final.

3. Función logística: que arranca de las necesidades de rapidez de la puesta a disposición del cliente y de menor coste posible, tiempo y costes a establecer en base a los conocimientos que se tengan sobre localización de los elementos que integran el producto final, proceso de transformación y reparto.

4. Función de producción:su responsabilidad consistirá en la integración de las diferentes personas que participan en todo el proceso, con el proyecto concreto, en base a los conocimientos que tenga sobre factores integradores, y prevención y corrección de los posibles factores desintegradores.

Como hemos observado, los conocimientos especializados y relacionados con el trabajo siguen siendo importantes, pero el énfasis principal ha cambiado. Existe una necesidad de

7 Véase Brunet y Morell (1998);Villa (1990)

8 Debemos destacar algunas de las diferencias que observan, entre la elaboración de un producto o un servicio. Alguna que podemos destacar son las competencias técnicas y sociales:conjunto de conocimientos y habilidades, conocimientos técnicos (objetos), conocimientos sociales (personas). En relación con el tiempo de trabajo: momento producción simultaneo al de consumo. Y en cuanto al control que se pueden ejercer a los trabajadores de servicios y de producción. 
cualificaciones extra-funcionales. Para Marion Hüchatermann (1997), son las cualificaciones esenciales no vinculadas a un determinado tipo de trabajo. Consideramos que los contenidos y usos de los nuevos requerimientos, tendrán que ver con las capacidades de los trabajadores de tipo comportamental. Pero, no sólo eso, sino que tendrían más relación con la simple adhesión a los criterios empresariales, al menos con los discursos sobre la competitividad y optimización de nuevos equipos o de nuevas técnicas organizativas ${ }^{9}$. Se trataría de poner más atención en el trabajo para controlar el rendimiento de las instalaciones, estar dispuesto a asumir tareas adicionales o en definitiva, ser un ser adaptable y todo ello, como ser reseña en el grupo de los trabajadores, sin ser reconocido formalmente.

No podemos olvidar, la importancia de la denominada sociedad de la información y de la comunicación, que como señala Cecilia Castaño (1997) es complementaria de un determinado tipo de mano de obra:cualificada, nivel de formación mayor, polivalente (capacidad de desarrollar diferentes tareas), capacidad de iniciativa y responsabilidad, transformaciones organizativas orientadas a la flexibilidad y la diferenciación del producto. Para Drucker (1993:199), será la sociedad del saber «en la sociedad del saber la gente tiene que aprender cómo aprender». Es más, puede que en la sociedad del saber las materias importen menos que la capacidad del estudiante para continuar aprendiendo y su motivación para hacerlo. La sociedad poscapitalista exige estudiar de por vida.

Por último, la posición que ocupa la empresa en el proceso global de elaboración de un bien o servicio, como señala Castillo (1989) construye los requerimientos cualificacionales. Intentamos ir un poco más lejos. Ya que lo sustancial es la posición de la empresa en el proceso y estructura de valorización de capital (elaboración de objetos de trabajo, medios de trabajo y medios de vida y empleo). 


\section{Bibliografía}

BAETGHE, M. y OBERBECK, H. (1995). El futuro de los emple ados. MTSS. Madrid.

BRUNET, I.y BELZUNEGUI, A.(2000). Empresa y estrategia en la perspectiva de la competencia global, Ariel Economía, Barcelona.

BRUNET, I. y MORELL, A. (1998). Clase, educación y trabajo. Madrid: Ed. Trotta

CASTILLO (1989). La división del trabajo entre empresas. MTSS. Madrid.

CASTAÑO, C. (1994). Tecnología, empleo y trabajo en España. Alianza Editorial.

CASTAÑO, C. (1997). «Transformaciones productivas y empleo. Importancia de la formación permanente», Revista del Ministerio de Trabajo y Asuntos Sociales, núm.1, pág. 89-101. CASTEL, R. (1997). La metamorfosis de la cuestión social, Paidos, Madrid.

CASTELL, M (1997). La era de la información. Economía, socie dad y cultura. Vol.I La sociedad red. Alianza editorial, Madrid. CENTRO ESTUDIOS ECONOMICOS FUNDACIÓN TOMILLO (1996). El empleo en España y Europa. Un análisis compa rado por sectores. Fundación Argentaria. Madrid.

DRUCKER, P. F. (1993). La sociedad poscapitalista, Apóstrofe, Madrid.

FINKEL, L. (1994). La organización social del trabajo. Pirámide. Madrid.

GORZ, A (1995). Metamorfosis del trabajo. Editorial Sistema. Madrid.

HOMS, O. y PRIETO, C.(1991). «La construcción social de los requerimientos cualificacionales en las empresas españolas», Revista de Economía y Sociología del Trabajo, Núm.1314/Septiembre- Diciembre, pág. 218-224.

HÜCHATERMANN, M (1997). «Preparación para las cualificaciones empresariales: una demanda de futuro», Situación, pág. 269-287

IBAÑEZ, J. (1979). Más allá de la sociología, Siglo XIX, Madrid. IESA (1987). Cambios de cualificación en las empresas espa ñolas. CSIC.Madrid. 
KERN, H. y SCHUMANN, M.(1989). El fin de la división de la di visión del trabajo. MTSS. Madrid.

LOPE, A. y MARTIN, A. (1993). "Cambio técnico y recualificación. Formación y adquisición de las cualificaciones en la empresa. Un estudio de casos.», Sociología del Trabajo, nueva época, núm. 19, otoño de 1993, pp 69-97.

LOPE, A. (1996). Innovación tecnológica y cualificación: la pola rización de las cualificaciones en la empresa, Consejo Económico y Social, Madrid

LOPE, LOZARES y MIGUELEZ (1997). «Perspectivas de análisis y primeros resultados de una investigación sobre la relación entre formación y empleo", REIS no 77-78, pp 263-305.

LLORENS, C., MARTÍ, J. y MIQUEL, J, (1996). «El debat sobre la formació», Revista Catalana de sociología, 2/96 pp. 51-82.

MARRERO RODRÍGUEZ, J.R. «Semejanzas y diferencias en la producción de bienes y servicios», Sociología del Trabajo, núm. 26.

MARTIN ARTILES, A. (1999). "Organización del trabajo y nuevas formas de gestión laboral», en Miguelez, F. y Prieto, C., Las relaciones de empleo en España, Siglo XXI, Madrid

QUIT (2000). ¿ Sirve la formación para el empleo?, CES, Madrid RECIO, A. (1997). Trabajo, personas y personas. Icaria. Fuhem.Madrid

RITZER (1993). La MacDonalización de la sociedad. Ariel Económica. Madrid.

SAEZ, F. -coord. (2000). Formación y empleo, Visor, Madrid.

SOLANS, J. R., PAC, D. y NAVARRETE, M. (1997). Estudio de colectivos con dificultad de acceso a la formación, Federación Minerometalurgiaca de CCOO, Zaragoza.

VILLA, PAOLA (1990). La estructuración de los mercados de tra bajo. La siderurgia y la construcción en Italia. Madrid: Ministerio de Trabajo y Seguridad Social. 